\title{
A Comparative Analysis of the Proteins and Lipids in Breast, Leg, and Thigh Meats of a Commercial Domesticated Turkey
}

\author{
W. Keith Ray, Sherry B. Hildreth, Judith M. Jervis and Richard F. Helm * \\ Department of Biochemistry, Virginia Tech, Blacksburg, VA 24061 \\ * Author of Correspondence: RF Helm (helmrf@vt.edu)
}

\begin{abstract}
A commercially reared domesticated turkey (Meleagris gallopavo) was purchased from a local market and sections of tissue representing leg, thigh, and breast were harvested and processed for analysis of the lipids and proteins present. Leg and thigh tissue was enriched in mitochondrial proteins whereas the breast tissue was enriched in glycolytic enzymes as well as the cytosolic and mitochondrial forms of glycerol-3-phosphate dehydrogenase. A potential marker for breast tissue muscle formation and/or function was also identified. The tissues could clearly be separated based upon their lipid profiles with little differences in cardiolipin levels suggesting that mitochondrial surface areas may be similar across the tissues. The most significant differences in the lipids were found to be higher levels of oxidized lipids in thigh meat. This work provides the first untargeted proteome and lipidome datasets for the domesticated turkey. The proteome dataset is accessible from ProteomeXchange Consortium via the PRIDE partner repository with the identifier PXD008207.
\end{abstract}

Keywords: glycolysis; lipidome; meat; mitochondria; oxidation; proteome; turkey 


\section{INTRODUCTION}

The yearly per capita consumption of domesticated turkey meat in the United States is over 7.5 $\mathrm{kg}$, with the industry rearing 233 million turkeys per year (National Turkey Federation). It is estimated that in the United States on Thanksgiving Day alone, approximately 46 million turkeys are cooked and consumed as a central part of the holiday. Considering the economic and societal importance of the domesticated turkey, these tissues have yet to be evaluated at the proteomic and lipidomic levels using untargeted/unbiased approaches to assess major differences. Such information is valuable for assessing turkey physiology, breeding efforts, and meat quality.

Untargeted proteomic studies attempt to assess the relative abundance of proteins across sample sets by using mass spectrometry signal intensities from ions derived from the proteins as a surrogate for relative amounts (Aebersold and Mann, 2016). This technique is generally performed by a "bottom-up" approach where isolated proteins are converted to peptides via a protease digestion. The peptides are then separated by liquid chromatography with the column eluent sprayed directly into a mass spectrometer, which assesses the peptides for properties (mass and fragmentation patterns). These data are then queried against a proteome database in order to both identify the peptides present and subsequently provide relative levels of the proteins across the samples. The availability of the domestic turkey genome (Dalloul et al., 2010) provides the ability to query the proteome of selected tissues in order to assess differences that relate to the physiology of the tissues investigated.

Lipidomic analyses also utilize LC-MS for measuring all ions present and subsequently analyze the data for significant differences (Knittelfelder et al., 2014). Sample preparation generally employs a liquid-liquid extraction of the tissues to enrich for the hydrophobic lipid fraction. The main difference between lipidomic and proteomic data analysis is that the lipids are characterized without matching to a database. Instead, features (ions) that differ in relative abundances across samples are identified based upon their retention time in the column along with their characteristic fragmentation features. Statistical analysis identifies the main features that are different between the samples, at which time efforts are made at identifying the lipids that differ significantly between samples. Lipid identification is challenging as these compounds range widely in concentration and have structural features that oftentimes prevent clear identifications (Koelmel et al., 2017).

Due to the societal and economic importance of the domesticated turkey, we sought to characterize the proteins and lipids present in a store-bought frozen turkey in order to assess the major differences in breast, leg, and thigh muscle tissue.

\section{MATERIALS AND METHODS}

General. All materials, unless otherwise specified, were obtained from Sigma-Aldrich and were of the highest available quality. Solvents, unless otherwise specified, were LC-MS grade and obtained from Spectrum Chemical.

Sample Preparation. A turkey $(6.1 \mathrm{~kg})$ purchased at a local grocery store was thawed $\left(4{ }^{\circ} \mathrm{C}, 3\right.$ days), at which time the plastic wrapping was removed and tissues were taken from the breast, thigh and leg regions (approximately $5 \mathrm{gms}$ each). The tissue isolates then were stored at $-80^{\circ} \mathrm{C}$ until further processing. The isolates were thawed on ice and rinsed thoroughly with ice cold 
phosphate-buffered saline (PBS). Samples were homogenized in $4 \mathrm{ml}$ cell lysis buffer $(50 \mathrm{mM}$ Tris-HCl (pH 7.5), $1 \mathrm{mM}$ EDTA, $150 \mathrm{mM} \mathrm{NaCl}, 0.1 \%(\mathrm{w} / \mathrm{v})$ SDS, 0.5\% (w/v) sodium deoxycholate, $1 \%(\mathrm{w} / \mathrm{v}) \mathrm{NP}-40)$. After homogenization, samples for protein analysis were subjected to three rounds of freeze/thaw (liquid nitrogen $/ 37^{\circ} \mathrm{C}$ ). The homogenization solution for all samples was then separated from remaining chunks and the solutions stored at $-80^{\circ} \mathrm{C}$. Protein concentrations were determined by tryptophan fluorescence using a SpectraMax M2 (Molecular Devices) using a commercial standard solution of bovine serum albumin (ThermoFisher) to generate a standard curve (Wisniewski and Gaugaz, 2015).

SDS-PAGE Analysis of the Protein Lysates. Aliquots of the samples containing 40 or $80 \mu \mathrm{g}$ protein were precipitated using methanol $(1 \mathrm{~mL})$ and storage over night at $-80^{\circ} \mathrm{C}$. Precipitated protein was recovered by centrifugation at room temperature for 15 minutes at $11000 \mathrm{x} \mathrm{g}$. Protein pellets were solubilized in $40 \mu \mathrm{l}$ Laemmli Sample Buffer (Bio-Rad) containing $50 \mathrm{mM}$ dithiothreitol (DTT) and incubated at $95^{\circ} \mathrm{C}$ for 15 minutes prior to loading onto a $10.5-14 \%$ acrylamide Tris-HCl gel (Bio-Rad/Criterion) along with molecular weight markers (Bio-Rad) and Tris/Glycine/SDS gel running buffer (Bio-Rad). The separation was performed at a constant voltage of $125 \mathrm{~V}$ until the dye front ran off the gel. Protein within the gel was visualized using Proto Blue Safe protein stain (National Diagnostics) and the stained gel was imaged using a ChemiDoc XRS using QuantityOne software (Bio-Rad). Selected bands were excised and the proteins identified using a 4800 MALDI Tof-Tof mass spectrometer (AB Sciex), queried against the database using the MASCOT search engine (Matrix Science).

LC-MS Analysis of the Protein Lysates. Aliquots of the samples for protein analysis containing $100 \mu \mathrm{g}$ protein were treated with $1 \mathrm{ml}$ methanol and stored over night at $-80^{\circ} \mathrm{C}$ to precipitate protein. Precipitated protein was recovered by centrifugation at room temperature for 15 minutes at $11000 \mathrm{x}$ g. Protein pellets were rinsed twice with $1 \mathrm{ml}$ cold methanol. The protein was then resuspended in freshly prepared $8 \mathrm{M}$ urea containing $100 \mathrm{mM}$ ammonium bicarbonate (AmBic) at a concentration of $0.5 \mathrm{mg} / \mathrm{mL}$. Disulfide bonds were reduced by incubation at $37^{\circ} \mathrm{C}$ for 1 hour in the presence of $4.5 \mathrm{mM}$ DTT. Free sulhydryls were then alkylated by incubation in the dark for 30 minutes in the presence of $10 \mathrm{mM}$ iodoacetamide (IAA). Unreacted IAA was quenched by the addition of $10 \mathrm{mM}$ DTT. Urea concentration was brought to $1.6 \mathrm{M}$ by the addition of $100 \mathrm{mM} \mathrm{AmBic}, 5 \mu \mathrm{g}$ trypsin was added and the digestion was carried out over night at $37^{\circ} \mathrm{C}$. Digestions were desalted using Oasis HLB $1 \mathrm{cc} 10 \mathrm{mg}$ extraction cartridges (Waters). Desalted peptides were dried using a vacuum concentrator and resuspended in $50 \mu 1$ water containing $0.2 \%(\mathrm{v} / \mathrm{v})$ formic acid.

The resulting samples were analyzed using an Orbitrap Fusion Lumos equipped with an Easy-nLC 1200 UPLC and an Easy Spray nanospray source (ThermoFisher). The column utilized for peptide separation was a PepMap RSLC C18 $2 \mu \mathrm{m} 100$ A $50 \mu \mathrm{m}$ x $15 \mathrm{~cm}$ (ThermoFisher) in line with an Acclaim PepMap 100 trapping column $(100 \mu \mathrm{m} \times 2 \mathrm{~cm})$. Analysis utilized a 110 minute gradient from 4 to $50 \%$ solvent B where A is $0.2 \%(\mathrm{v} / \mathrm{v})$ formic acid in water and $\mathrm{B}$ is 20:80 water:acetonitrile containing $0.2 \%(\mathrm{v} / \mathrm{v})$ formic acid. Column temperature was maintained at $55^{\circ} \mathrm{C}$ and the ion transfer tube at $275^{\circ} \mathrm{C}$. Other analysis conditions were as follows: $2 \mu \mathrm{l}$ injection, $300 \mathrm{nl} / \mathrm{min}$ flow rate, $2300 \mathrm{~V}$ ion spray voltage, sweep gas at 7, MS scans utilizing the orbitrap at 60,000 resolution for m/z 300-1350, an AGC target of $1 \mathrm{e} 6$ with $50 \mathrm{~ms}$ max inject time, profile, positive, MSMS targets chosen using MIPS looking for peptide-like isotopic distribution with a $z=2-6$ and a minimum intensity of $2 \mathrm{e} 3$, 
dynamic exclusion for $5 \mathrm{sec}$, priority given to the most intense with a max cycle time of $2 \mathrm{sec}$ (Top Speed mode), MSMS isolation window of $0.7 \mathrm{~m} / \mathrm{z}, \mathrm{HCD}$ at 30, ion trap turbo scan detection for $\mathrm{m} / \mathrm{z}$ 200-1200, AGC target of $4 \mathrm{e} 4$ with a max inject time of $15 \mathrm{~ms}$, centroid.

Proteome Data Analysis. Mass spectrometry data were analyzed using Proteome Discoverer (Version 2.2.0.388, ThermoFisher). Masses were first recalibrated by the Spectrum Files RC node using a quick search followed by a thorough search using Sequest HT of Version 5.0 of the turkey proteome available for download at the NCBI FTP website. A contaminants database (ThermoFisher) along with a reverse decoy database were added to the search for identification of contaminants as well as for determining false discovery rates. To minimize missing values, identified peptide peaks were matched among runs with unidentified peaks using a combination of the Minora Feature Detector, Feature Mapper and Precursor Ions Quantifier nodes. Data visualization was aided by the Perseus software package (Tyanova et al., 2016).

Lipid Sample Preparation. The methods employed were essentially a combination of previously published methods (Coman et al., 2016; Matyash et al., 2008). Tissues for lipid analysis were homogenized in $0.1 \%(\mathrm{w} / \mathrm{v})$ ammonium acetate $(25 \mathrm{~mL})$ using a VWR power 200 homogenizer and an aliquot $(3 \mathrm{~mL})$ was transferred to a clean amber glass vial and taken to dryness via lyophilization. All subsequent procedures were essentially that of Matyash and coworkers (PMID: 18281723). The dried samples were suspended in $\mathrm{MeOH}$ (1.5 mL) sealed with a teflon-lined screw cap and vortexed to suspend the solids and subsequently sonicated in a water bath to disrupt the materials as much as possible. Methyl tert-butyl ether (MTBE) was then added $(5 \mathrm{ml})$ and the mixture was extracted for $1 \mathrm{~h}$ at room temperature with occasional vortexing. Water was then added $(1.25 \mathrm{~mL})$ and the solution was mixed vigorously by hand and then allowed to settle. An aliquot $(1 \mathrm{~mL})$ was removed from the upper phase and taken to dryness, first under as stream of nitrogen to remove most of the MTBE, and then on a high vacuum line. The samples were resuspended in $\mathrm{CH}_{2} \mathrm{Cl}_{2} /$ methanol/water $(60: 30: 4.5,100 \mathrm{uL})$, centrifuged from $10 \mathrm{~min}(13 \mathrm{k} \mathrm{x} \mathrm{g})$ and an aliquot $(80 \mathrm{uL})$ transferred to an LC-MS vial for analysis.

Lipid Analysis. An Aquity I-class UPLC (Waters, Milford, MA) was directly interfaced with a Synapt G2-S mass spectrometer (Waters, Milford, MA). Lipid extracts $(5 \mu \mathrm{L})$ were injected onto an Acquity HSS T3 column $\left(1.8 \mu \mathrm{m}\right.$ particle $100 \times 2.1 \mathrm{~mm}$ id) kept at $55^{\circ} \mathrm{C}$ and operating at a flow rate of $400 \mu \mathrm{l} /$ minute. Mobile phase A was acetonitrile and water $(40: 60, \mathrm{v} / \mathrm{v})$ with $10 \mathrm{mM}$ ammonium acetate and mobile phase B was acetonitrile and isopropanol (10:90) containing 10 $\mathrm{mM}$ ammonium acetate. The initial conditions of $60 \% \mathrm{~A}$ and $40 \% \mathrm{~B}$ was followed by a linear gradient for 10 minutes to $100 \% \mathrm{~B}$. The gradient was held at $100 \% \mathrm{~B}$ for 2 minutes after which the gradient returned to original start conditions for equilibration (4 min, $16 \mathrm{~min}$ run time). Sample analysis were performed in triplicate in both positive and negative ion modes using the high-resolution instrument configuration, with data collected in centroid mode.

Negative mode analysis was carried out using the following settings: capillary voltage 2.2 , scan range $50-1800 \mathrm{~m} / \mathrm{z}$, source temperature $150^{\circ} \mathrm{C}$, cone voltage 30 , source offset 80 , desolvation temperature $400^{\circ} \mathrm{C}$, cone gas flow $50 \mathrm{~L} / \mathrm{hr}$, desolvation gas flow $500 \mathrm{~L} / \mathrm{hr}$ and nebulizer gas flow 6. Positive mode analysis was carried out using the following settings: capillary voltage 3.2 , scan range $50-1800 \mathrm{~m} / \mathrm{z}$, source temperature $150^{\circ} \mathrm{C}$, cone voltage 30 , source offset 80 , desolvation temperature $400^{\circ} \mathrm{C}$, cone gas flow $50 \mathrm{~L} / \mathrm{hr}$, desolvation gas flow 
$500 \mathrm{~L} / \mathrm{hr}$ and nebulizer gas flow 6. Leucine enkephephalin was used as the internal mass reference. The cycle time was 0.2 seconds with a lock mass scan every 20 seconds. MS/MS data was collected using the source conditions listed above with collision energy added to induce fragment formation. Additionally, master mix samples were analyzed in $\mathrm{MS}^{\mathrm{E}}$ mode which allowed simultaneous collection of MS data and fragmentation data.

The data were processed using MarkerLynx XS (Waters, Milford, MA) for feature discovery and statistical analysis. Negative mode processing returned 158 features while positive mode returned 741 features; both sets used the parameters of 250-1200 mass range, marker intensity threshold of 2500 and automated deisotoping. Pairwise comparisons of features detected in each tissue type were used to identify features that were different among the tissue types. Feature identification was done using a combination of MS/MS fragmentation analysis, $\mathrm{MS}^{\mathrm{E}}$ data and exact mass. The LIPID MAPS database and literature were used to assign lipid IDs (Fahy et al., 2007).

\section{RESULTS AND DISCUSSION}

Tissues from the purchased turkey were prepared for analysis as described in Figure 1. An initial assessment of the most abundant proteins in the tissues was performed using SDS-PAGE at two different loading levels (Figure 2). Bands were selected for identification via MALDI-TOF/TOF mass spectrometry with the identifications shown. At the level of SDS-PAGE, the banding patterns of leg and thigh proteins are more similar to one another than the breast tissue proteins. There are also several discrepancies between the observed mass of the proteins by SDS-PAGE and the calculated mass based upon the proteome database used, Version 5.0 of the turkey proteome, available for download at the NCBI FTP website (GCF_000146605.2). For example, fructose bisphosphate aldolase was identified in both Bands 5 and 6 , which corresponds to an apparent mass of approximately $35 \mathrm{kDa}$. However, the calculated mass based upon the database was determined to be $8.3 \mathrm{kDa}$, well below the established masses of most reviewed aldolases in the UniProt database, which is approximately $39 \mathrm{kDa}$. This discrepancy is related to the process by which the proteome database is generated, where protein coding sequences are generated computationally. The gene models employed in generating the protein databases are oftentimes not completely accurate, leading to discrepancies such as the one described here. Clarification of the true protein and gene sequences can be accomplished through proteogenomics approaches, where genomic, transcriptomic and proteomic data are interrogated together (Nesvizhskii, 2014). Future work in the area of turkey genomics and proteomics will benefit greatly from work in this area.



Figure 1. Preparation of Samples for Lipid and Protein Analyses. A frozen bird was thawed and longitudinal slices were taken. Samples were homogenized and separated into aliquots for protein and lipid analyses using standard techniques. See Materials and Methods for details. 


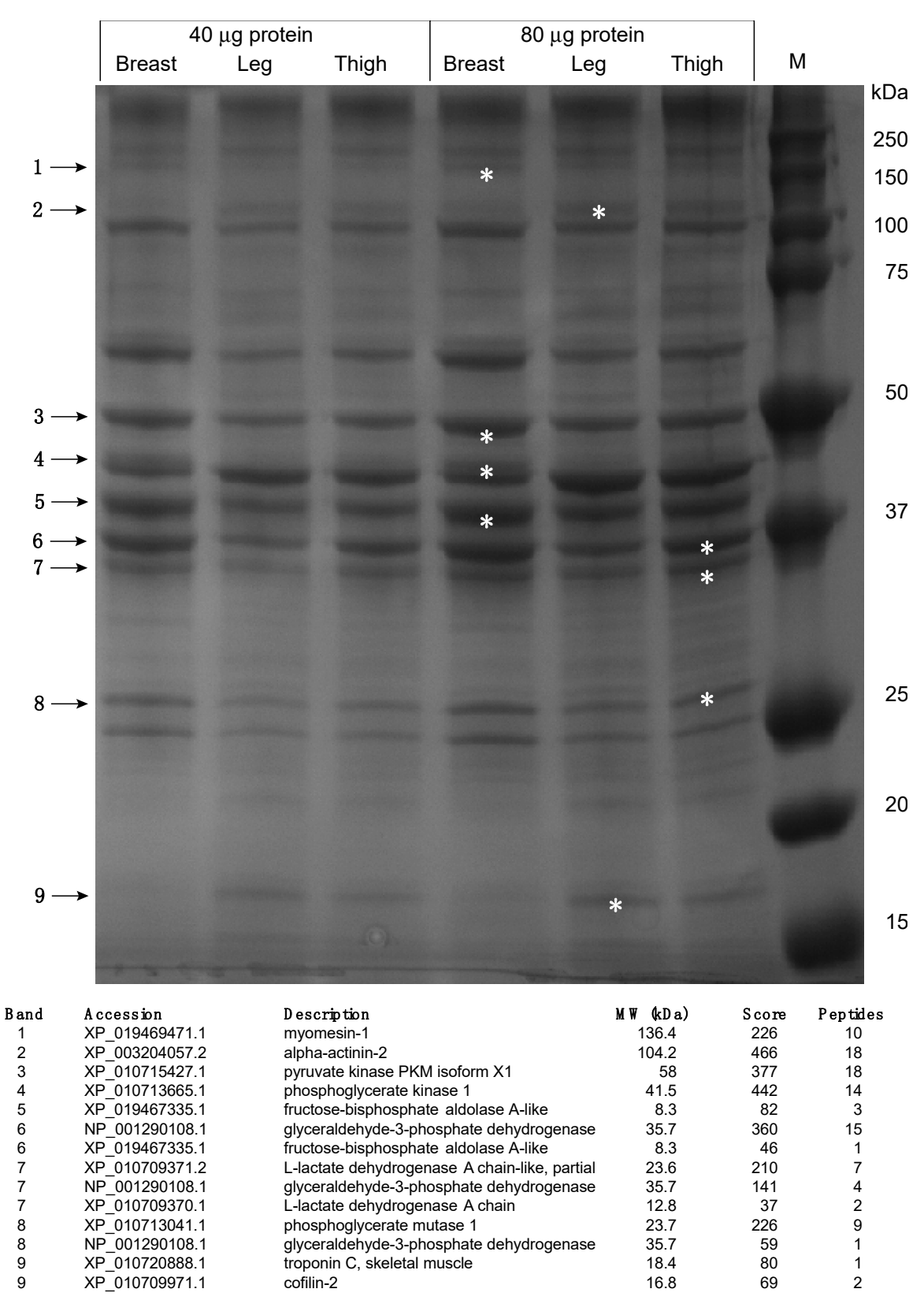

Figure 2. SDS-PAGE Separation and MALDI-TOF/TOF Analysis of the Breast, Leg and Thigh Protein Lysates. Selected bands (asterisks) were excised from the gel and processed to peptides for identification via MALDITOF/TOF and the MASCOT search engine.

Proteomic analyses via bottom-up LC-MS analyses can provide a higher level of coverage than what can be obtained by SDS-PAGE methodologies, with relative quantification utilizing label-free techniques permitting direct comparison between sample types. There were slightly over 400 proteins quantified for comparison of relative levels between tissues using a scaled abundance approach (Supplemental Table S1). As with the MALDI-based identifications, protein identifications and quantifications are based on NCBI proteome database. A direct comparison of relative levels in the form of a heat map is shown in Figure 3. Based upon the 
heat map as well as Principal Components Analysis, the leg and thigh tissues are more similar to one another that the breast tissue, confirming the relative abundances found by SDS-PAGE protein staining differences. Clustering analysis based on relative abundances using the Perseus software package on the proteins shown in Table S1 separated the proteins into 3 main categories, as indicated on the heat map (Figure 3), those in higher abundance in breast tissue (40 proteins, Cluster A), those higher in leg tissue (29 proteins, Cluster B), and those higher in both leg and thigh (288 proteins, Cluster C).

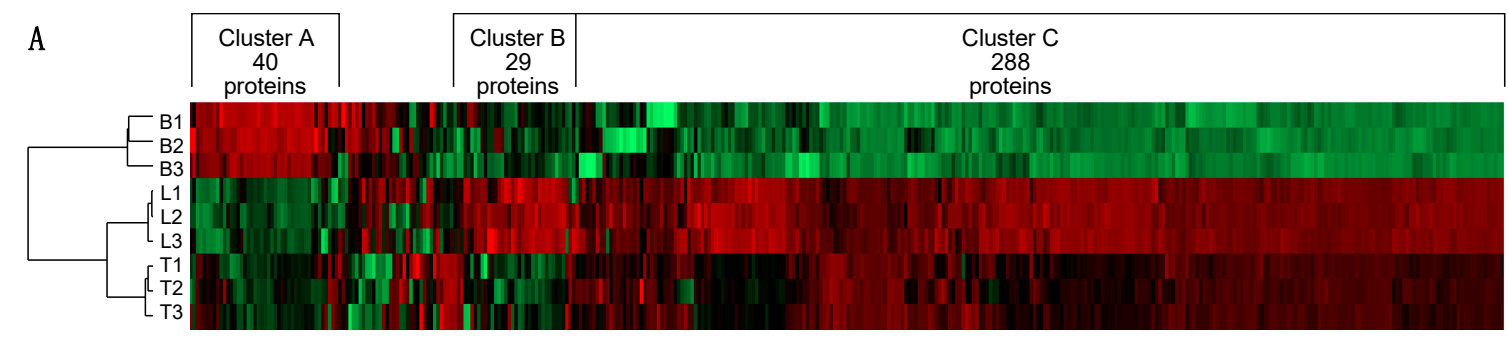

B

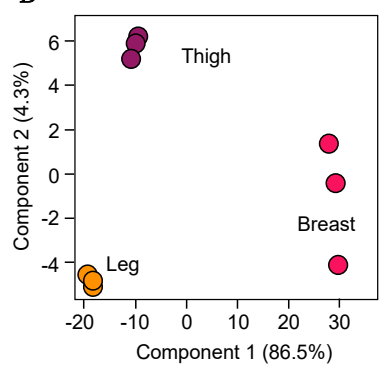

Figure 3. Overview of the Proteomic Analysis. A, Heat map of the quantified proteins, clustered based upon relative abundances across the three tissue types (B, breast; $L$, leg; $T$, thigh). B, Principal components analysis indicating that over $85 \%$ of the differences are in Component 1.

Breast tissue prefers glycolysis but still need functional mitochondria. The proteins found in higher abundance in breast tissue were clearly enriched in enzymes related to carbohydrate metabolism, ranging from glycogen synthesis (glycogenin) and degradation (glycogen phosphorylase) through almost the entire glycolytic pathway, with the only proteins absent being hexokinase and phosphofructokinase (Table 1). Fully 23 of the 40 proteins in this category were related to cytosolic/glycolytic energy production. The cytosolic and mitochondrial forms of glycerol-3-phosphate dehydrogenase are well established markers of muscles associated with winged flight (Mracek et al., 2013); which is the main role of breast tissue in birds. The two enzymes work in tandem but in opposite directions in order to shuttle electrons into the mitochondrial electron transport chain from the cytosol. This process delivers electrons to the ubiquinol pool in return for replenishing the cytosolic supplies of $\mathrm{NAD}^{+}$, which is required by glyceraldehyde-3-phosphate dehydrogenase for continued glycolytic energy production. Thus, while breast meat is considered to be relatively low in mitochondrial content, there is clear evidence of a highly abundant mitochondrial protein present in turkey breast meat.

Potential Biomarkers of Breast Tissue Physiology. While the turkey industry has made tremendous strides in breeding to improve breast tissue muscling, this phenotype can also lead to the development of pale, soft, and exudative (PSE) meat, which results in, among other things, excessive water loss during cooking (Strasburg and Chiang, 2009). Several studies have suggested that, similar to pork, post-mortem calcium levels are associated with PSE, with the ryanodine receptors implicated (Desai et al., 2016; Schilling et al., 2017; Strasburg and Chiang, 
2009 ). The levels of ryanodine receptor 3 ( $\beta$ RyR) as well as a ryanodine receptor 3-like protein (XP_010709934.1) were found to be significantly higher in the breast tissue versus leg and thigh (Figure 4). As these two proteins were found in abundance, a targeted proteomics assay could be developed to monitor this protein for assessing its contribution to PSE and/or breast meat quality (Patterson et al., 2017).

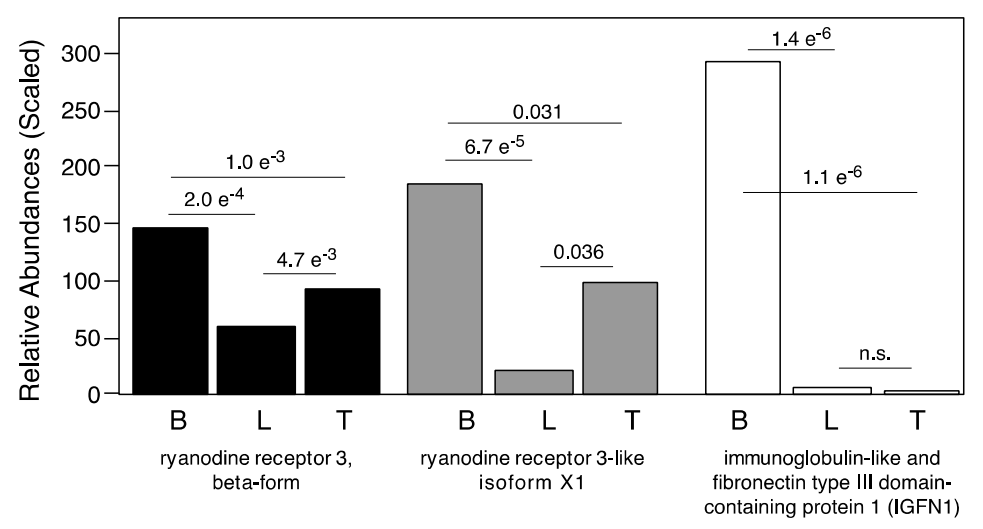

Figure 4. Potential Biomarkers of Breast Tissue Physiology. The abundances of the ryanodine receptors identified along with the turkey IGFN1 proteins are shown along with their associated $p$-values for pairwise comparisons.

Another protein found almost exclusively in breast tissue is presently annotated as immunoglobulin-like and fibronectin type III domain-containing protein 1 (XP_010722346.1; Figure 4, Supplemental Table S1). A BLAST search of this protein matches it most closely with birds with similar habits to turkeys (chicken, guineafowl, penguin). This protein in humans (IGFN1) is thought to interact with at least two different proteins, eukaryotic translation elongation factor 1 alpha 2 (EEF1A2) and KY (kyphoscoliosis peptidase). Both proteins have been found to be upregulated in mouse models of increased muscling (Barbe et al., 2017; Miao et al., 2015), suggesting that the turkey IGFN1 may play a crucial role, and hence a marker, for breast tissue muscling in poultry.

Proteins enriched in leg tissue are a mixed bag of fundamental processes. Unlike the breast tissue, which was enriched in proteins related to carbohydrate metabolism, the proteins more highly abundant in leg tissues covered a broad swath of biochemical processes (Table 2) including muscle and calcium-based processes. Several were mitochondrial with three of them related to $\mathrm{NAD}^{+} / \mathrm{NADH}$ levels. The fact that both histone $\mathrm{H3}$ and a regulator of histone methylation (SMYD1) were enriched in leg tissues suggests there may be some key histonebased post-translational modifications that differentiate leg from breast and thigh tissue.

Most proteins identified were higher in leg and thigh relative to breast tissue. The heat map as well as the PCA plot shown in Figure 3 indicate that the leg and thigh tissues are more like each other than breast tissue. Mitochondrial enzymes comprised about $25 \%$ of Cluster $\mathrm{C}$ in the heat map (70/288), and included the enzymes associated with pyruvate dehydrogenase/TCA cycle, fatty acid $\beta$-oxidation, and oxidative phosphorylation (Supplementary Table S2). A list of the 15 most significantly different proteins based upon their summed PEP scores (relative confidence level for identification) is shown in Figure 5. Considering the high levels of 
mitochondrial proteins in leg and thigh tissue, it is not surprising that myoglobin, the protein most closely linked to meat color was found in trace amounts in breast tissue and was highest in leg. Interestingly, leg and thigh tissues are much higher in lactate dehydrogenase (LDH, B-form) than breast tissue, even though breast tissue is more glycolytic, a process that typically requires lactate formation and subsequent excretion. It may be that the glycerol-3-phosphate shuttle is more important than lactate release in maintaining cytosol $\mathrm{NAD}^{+} / \mathrm{NADH}$ ratios in breast tissue, suggesting functional mitochondria are present in breast tissue, even though they have relatively low levels of the oxygen storage protein, myoglobin.

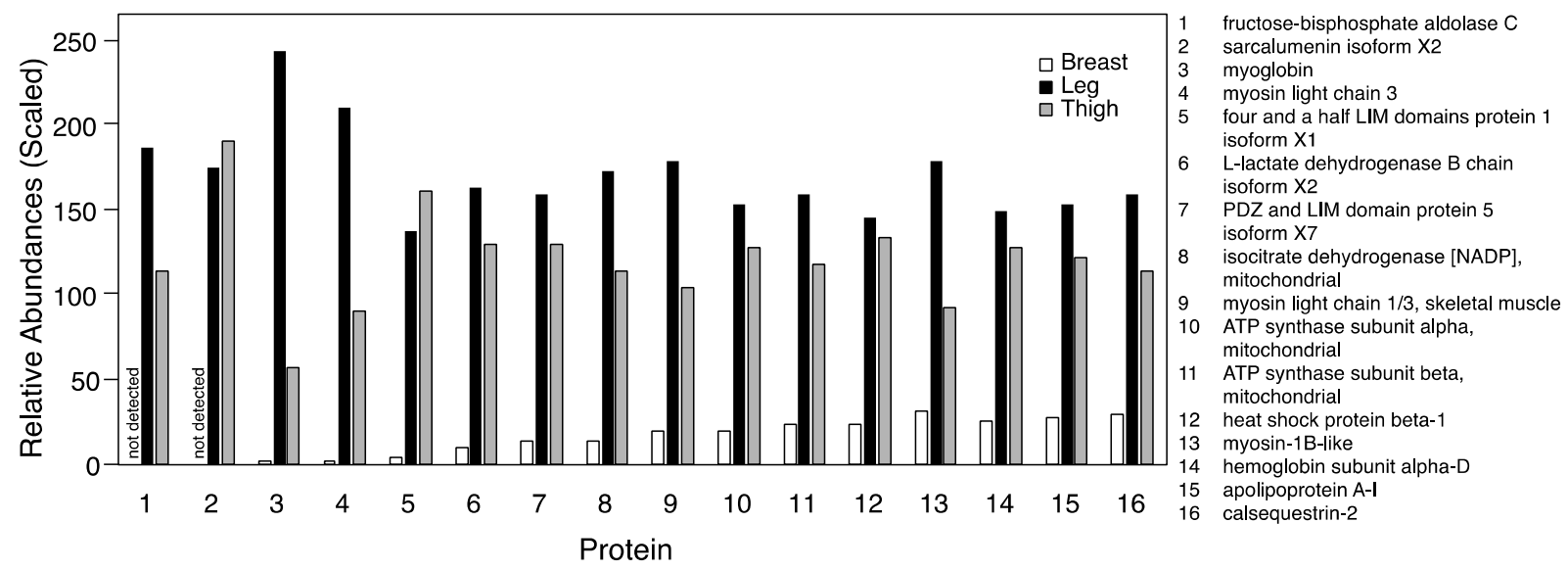

Figure 5. Selected Proteins with Significantly High Leg:Breast Protein Abundance Ratios. Proteins are shown (1-16) in order of decreasing Leg:Breast ratios, with the levels of Proteins 1 and 2 below the limit of detection for the samples analyzed. Also see Table S2.

Lipid Analyses Reveal Differences in Levels of Oxidation. The lipid profiles for the tissues were compared against one another along with a "master mix" of the three samples combined into one. Principal Components Analyses of both the positive and negative ionization mode samples revealed a clear separation, with the master mix showing a combination of all three (Figure 6). Efforts to determine the lipids that resulted in the class separation observed led to the finding that thigh muscle was more prone to oxidation than that of breast and leg. A recent study of frozen turkey breast and thigh meats also reported a higher oxidation potential of thigh over breast tissue (Juskiewicz et al., 2017). Interestingly, when we manually searched for the lipid mitochondrial marker, cardiolipin, three different forms were identified, but the levels were not significantly different between tissues. This would imply that the relative surface areas of mitochondria between tissues are relatively the same. However, the proteomics work clearly indicated more mitochondrial proteins in both leg and thigh meat relative to breast meat. Further work in the area of mitochondrial dynamics and function between tissues may provide insights into turkey physiology and meat science. 
A Positive lon Mode

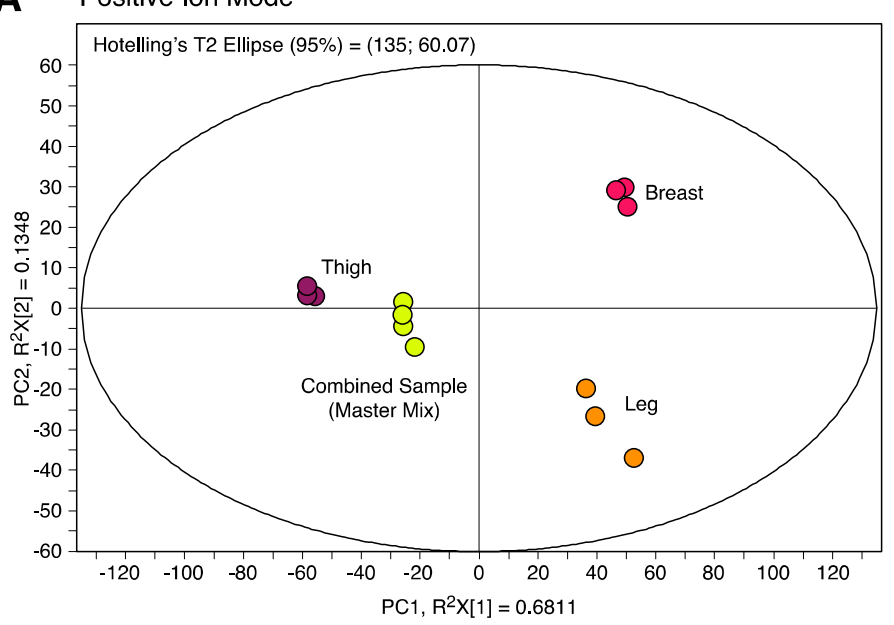

B Negative lon Mode

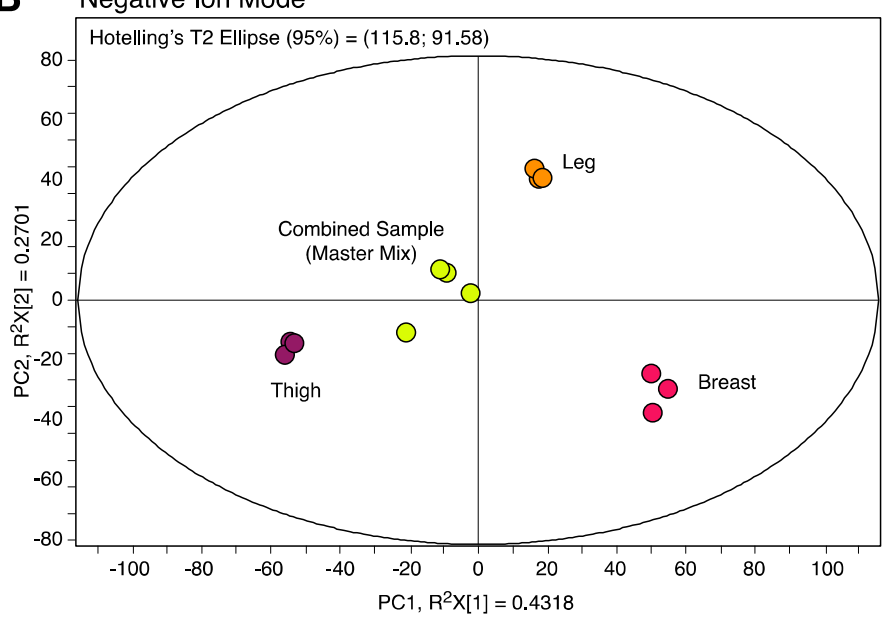

Glycerophospholipids and Unknowns

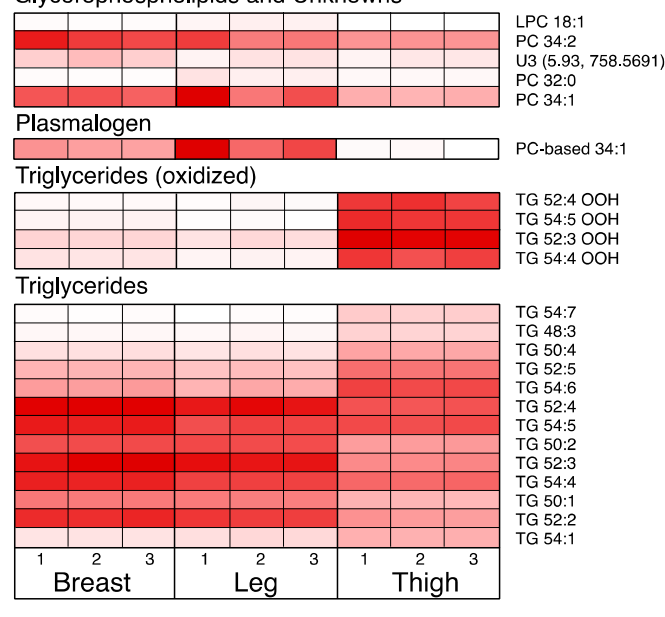

Fatty Acids

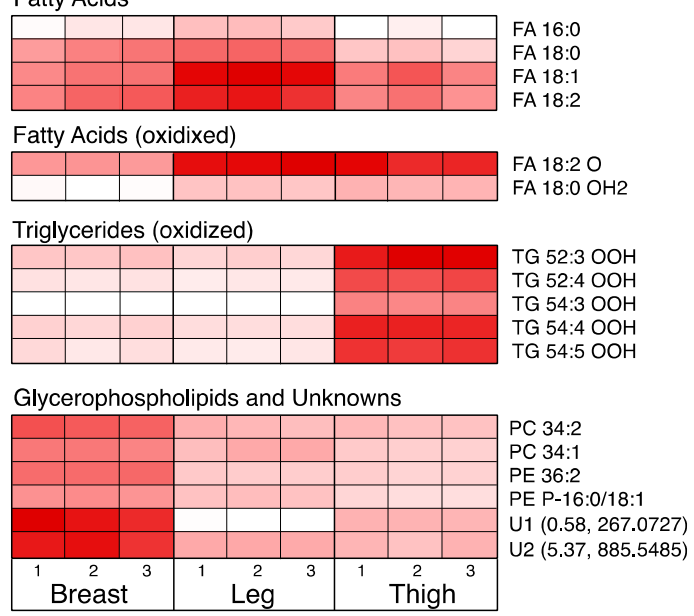

Figure 3. Untargeted Lipid Analysis of the Breast, Leg and Thigh Tissues. A, Principal Components Analysis of the positive ion mode analyses along the metabolites that changed the most based upon tissue (lower levels are white, higher levels are burgundy. B, Principal Components Analysis of the negative ion mode analyses along the metabolites that changed the most based upon tissue (lower levels are white, higher levels are burgundy. Abbreviations: LPC, lysophosphatidylcholine; PC, phosphatidylcholine; PE, phosphatidylethanolamine; PE-P, ethanolamine-based plasmalogen; $U$, Unknown. Unknowns are listed with retention time $(\mathrm{min})$ and $\mathrm{m} / \mathrm{z}$.

\section{SUMMARY}

The work presented here provides the first assessment of the proteomes and lipidomes of breast, leg and thigh tissues of a domestic turkey. Differences in protein abundances mirror the level of physical activity for the bird, with leg tissues having higher levels of mitochondrial proteins relative to thigh and breast meat. Several potential biomarkers of breast tissue were identified that may aid further research in turkey growth, physiology, and post-mortem biochemistry. Lipids appear to oxidize faster in thigh tissues, and based upon cardiolipin levels, total mitochondrial surface areas between tissues are similar. 


\section{ACKNOWLEDGEMENTS}

The mass spectrometry facilities used in this work are maintained with support of Virginia Tech and the Fralin Life Science Institute. This work was done, in part, as an exercise in providing undergraduate students in a general biochemistry class (BCHM 4115, Fall 2017) with hands-on training in working with real biological datasets. A full list of students that contributed to this work can be found in the below.

Contributions. RF Helm conceived the idea, analyzed the data and drafted the manuscript. WK Ray prepared and analyzed the proteome data, SB Hildreth analyzed the lipidomic data, and JM Jervis prepared the lipid samples. BCHM4115 Class analyzed the proteome data. A full list of student participants is shown below in alphabetical order. Those that contributed more in terms of analyses and forwarding the project are indicated in bold:

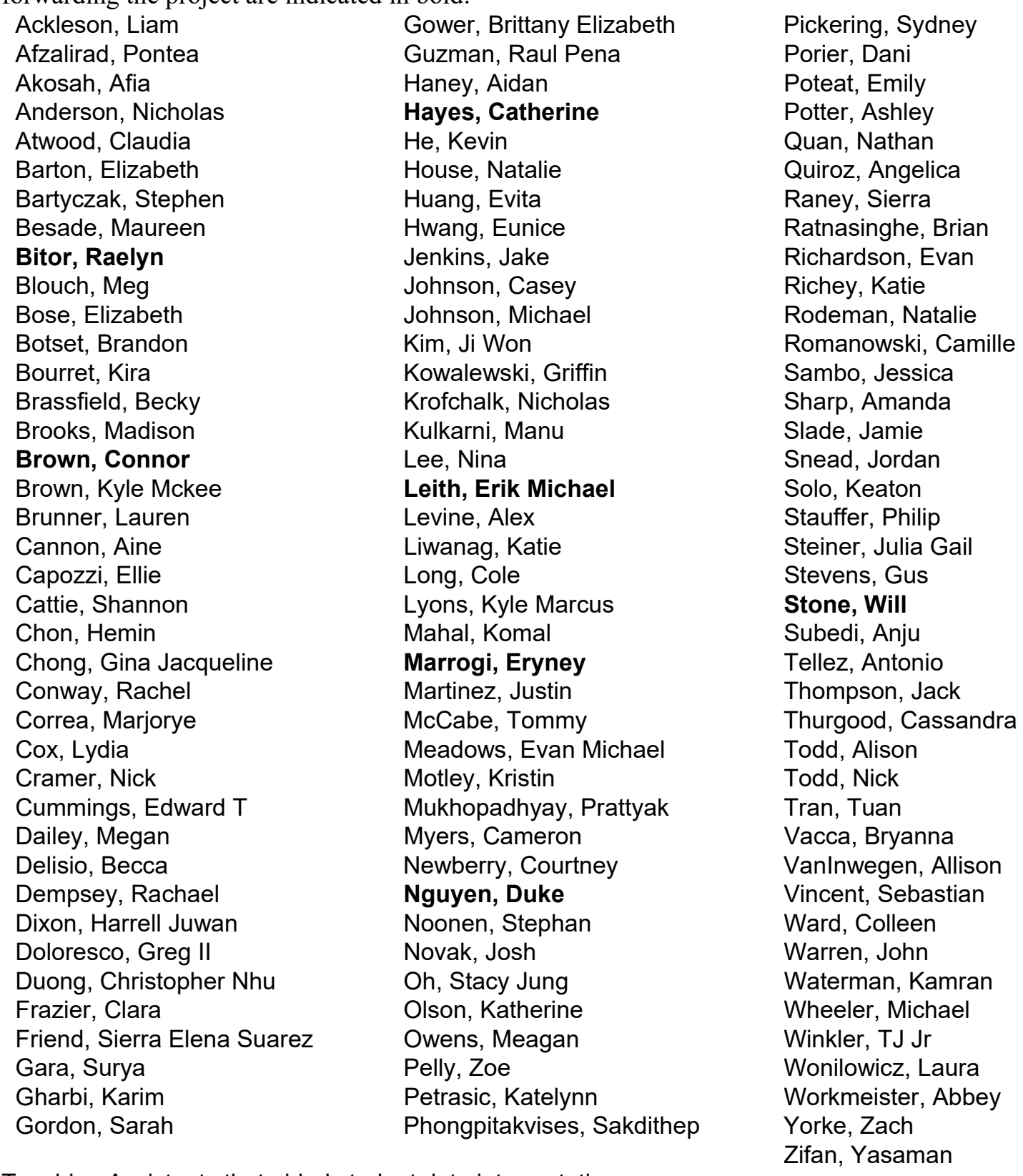

Teaching Assistants that aided student data interpretation:

Compton, Austin Cridland, Caitlin James, Hannah McCluskey, Anna Ngo, Nam 


\section{Literature Cited}

Aebersold, R., and Mann, M. (2016). Mass-spectrometric exploration of proteome structure and function. Nature 537, 347-355.

Barbe, C., Bray, F., Gueugneau, M., Devassine, S., Lause, P., Tokarski, C., Rolando, C., and Thissen, J.P. (2017). Comparative Proteomic and Transcriptomic Analysis of Follistatin-Induced Skeletal Muscle Hypertrophy. J Proteome Res 16, 3477-3490.

Coman, C., Solari, F.A., Hentschel, A., Sickmann, A., Zahedi, R.P., and Ahrends, R. (2016). Simultaneous Metabolite, Protein, Lipid Extraction (SIMPLEX): A Combinatorial Multimolecular Omics Approach for Systems Biology. Mol Cell Proteomics 15, 1453-1466.

Dalloul, R.A., Long, J.A., Zimin, A.V., Aslam, L., Beal, K., Blomberg Le, A., Bouffard, P., Burt, D.W., Crasta, O., Crooijmans, R.P., et al. (2010). Multi-platform next-generation sequencing of the domestic turkey (Meleagris gallopavo): genome assembly and analysis. PLoS Biol 8.

Desai, M.A., Jackson, V., Zhai, W., Suman, S.P., Nair, M.N., Beach, C.M., and Schilling, M.W. (2016). Proteome basis of pale, soft, and exudative-like (PSE-like) broiler breast (Pectoralis major) meat. Poult Sci 95, 2696-2706.

Fahy, E., Sud, M., Cotter, D., and Subramaniam, S. (2007). LIPID MAPS online tools for lipid research. Nucleic Acids Res 35, W606-612.

Juskiewicz, J., Jankowski, J., Zielinski, H., Zdunczyk, Z., Mikulski, D., Antoszkiewicz, Z., Kosmala, M., and Zdunczyk, P. (2017). The Fatty Acid Profile and Oxidative Stability of Meat from Turkeys Fed Diets Enriched with n-3 Polyunsaturated Fatty Acids and Dried Fruit Pomaces as a Source of Polyphenols. PLoS One 12, e0170074.

Knittelfelder, O.L., Weberhofer, B.P., Eichmann, T.O., Kohlwein, S.D., and Rechberger, G.N. (2014). A versatile ultra-high performance LC-MS method for lipid profiling. J Chromatogr B Analyt Technol Biomed Life Sci 951-952, 119-128.

Koelmel, J.P., Kroeger, N.M., Gill, E.L., Ulmer, C.Z., Bowden, J.A., Patterson, R.E., Yost, R.A., and Garrett, T.J. (2017). Expanding Lipidome Coverage Using LC-MS/MS Data-Dependent Acquisition with Automated Exclusion List Generation. J Am Soc Mass Spectrom 28, 908-917.

Matyash, V., Liebisch, G., Kurzchalia, T.V., Shevchenko, A., and Schwudke, D. (2008). Lipid extraction by methyl-tert-butyl ether for high-throughput lipidomics. J Lipid Res 49, 1137-1146.

Miao, Y., Yang, J., Xu, Z., Jing, L., Zhao, S., and Li, X. (2015). RNA sequencing identifies upregulated kyphoscoliosis peptidase and phosphatidic acid signaling pathways in muscle hypertrophy generated by transgenic expression of myostatin propeptide. Int J Mol Sci 16, 79767994.

Mracek, T., Drahota, Z., and Houstek, J. (2013). The function and the role of the mitochondrial glycerol-3-phosphate dehydrogenase in mammalian tissues. Biochim Biophys Acta 1827, 401410.

Nesvizhskii, A.I. (2014). Proteogenomics: concepts, applications and computational strategies. Nat Methods 11, 1114-1125.

Patterson, B.A., Matarneh, S.K., Stufft, K.M., England, E.M., Scheffler, T.L., Preisser, R.H., Shi, H., Stewart, E.C., Eilert, S., and Gerrard, D.E. (2017). Pectoralis major muscle of turkey displays divergent function as correlated with meat quality. Poult Sci 96, 1492-1503.

Schilling, M.W., Suman, S.P., Zhang, X., Nair, M.N., Desai, M.A., Cai, K., Ciaramella, M.A., and Allen, P.J. (2017). Proteomic approach to characterize biochemistry of meat quality defects. Meat Sci $132,131-138$.

Strasburg, G.M., and Chiang, W. (2009). Pale, soft, exudative turkey--The role of ryanodine receptor variation in meat quality. Poult Sci 88, 1497-1505.

Tyanova, S., Temu, T., Sinitcyn, P., Carlson, A., Hein, M.Y., Geiger, T., Mann, M., and Cox, J. (2016). The Perseus computational platform for comprehensive analysis of (prote)omics data. Nat Methods 13, 731-740.

Wisniewski, J.R., and Gaugaz, F.Z. (2015). Fast and sensitive total protein and Peptide assays for proteomic analysis. Anal Chem 87, 4110-4116. 